\title{
Effect of Pre-training and Post-training Nordic Exercise on Hamstring Injury Prevention, Recurrence, and Severity in Soccer Players
}

\author{
Ahmed Ebrahim Elerian, MD, $\mathrm{PhD}^{1}$, Mohsen M. El-Sayyad, MD, $\mathrm{PhD}^{1}$, Hend Adel Abdellhalim Dorgham, $\mathrm{BS}^{2}$
}

${ }^{1}$ Department of Basic Science, Faculty of Physical Therapy, Cairo University, Cairo;

${ }^{2}$ Department of Physical Therapy, Alexandria General Hospital, Alexandria, Egypt

Objective To investigate the effect of adding Nordic exercise as post-training in decreasing hamstring initial, recurrent injuries rates, and their severity.

Methods In this randomly controlled trial study, 34 professional football players aged 21 to 35 years were randomly assigned into two groups (17 players each) from Sporting clubs at Alexandria, Egypt. For group one, Nordic hamstring exercise (NHE) was performed pre-training and post-training. For group two, NHE was only performed pre-training. The control group was the same team during the previous season. Length of the trial was 12 weeks. The Australian football association injury form was used to collect incidence of injuries for each subject in both groups.

Results Pooled results based on total injuries showed that group one had significantly less hamstring initial injuries (92\% less) than the previous season, while group two had $80 \%$ less initial injuries and $85 \%$ less recurrent injuries than previous season. Regarding the severity of injuries in term of mean number of absent days, it was 1 day for group one and 2.7 days for group two while it was 7.95 days for the previous season during total risk time of $116.3 \pm 13.2$ and 117.6 \pm 5.7 exposure hours for group one and group two, respectively.

Conclusion The use of NHE as a prevention protocol was effective in reducing all hamstring injuries with the use of NHE during pre-training and post-training having the greatest effect.

Keywords Hamstring muscles, Leg injuries, Soccer, Egypt

\section{INTRODUCTION}

Hamstring strain injury is the most common noncon- tact muscle injury for soccer players, representing twothirds of thigh muscle strains [1]. Hamstring strain injury and recurrence rate were high in the past three decades.

Received January 22, 2019; Accepted February 28, 2019

Corresponding author: Ahmed Ebrahim Elerian

Department of Basic Science, Faculty of Physical Therapy, Cairo University, No. 77 street El-Maadi, Cairo, Egypt. Tel: $+20-220111675233$, Fax: +20 227294207, E-mail: dr_ahmed_elerian77@cu.edu.eg

ORCID: Ahmed Ebrahim Elerian (https://orcid.org/0000-0003-3335-8933); Mohsen M. El-Sayyad (https://orcid.org/0000-0002-2230-3067); Hend Adel Abdelhalim Dorgham (https://orcid.org/0000-0001-7245-5336).

(c) This is an open-access article distributed under the terms of the Creative Commons Attribution Non-Commercial License (http://creativecommons.org/ licenses/by-nc/4.0) which permits unrestricted noncommercial use, distribution, and reproduction in any medium, provided the original work is properly cited. Copyright ( 2019 by Korean Academy of Rehabilitation Medicine 
They occur mainly before the end of each half game when fatigue plays a role [2]. Hamstring strain injury could lead to inability to play for cup up to 90 days [3]. This, it can have a great impact on players and the team [4]. Nordic hamstring exercise (NHE) is an eccentric strengthening exercise. It is one of the most important components of the FIFA 11+ (Fédération Internationale de Football Association) exercise program. It has been proven that NHE can decrease hamstring injuries by $51 \%$ among soccer players per 1,000 hours of exposure [5]. Such effect of NHE could due to increased muscle activation and a shift in optimum torque production towards longer lengths, leading to 'injury-protective' effect of NHE. Induced muscle damage can lead to sarcomerogenesis (increased muscle fiber length), allowing it to stretch further. Only one NHE training session can cause immediate shift $\left(7.7^{\circ}\right)$ in optimum knee flexion torque. Such effect can be sustained at 10 days after NHE [6]. NHE for 10 weeks can lead to $20.8 \%$ fascicle length increase [7].

The percentage of injury prevention risk varies, meaning the level of effectiveness is not the same. After applying FIFA $11+$ prevention program, $20 \%-50 \%$ reduction in injury risk has been reported [8]. High compliance to FIFA $11+$ prevention program has led to significant decreased in injury risk (by 57\%) [9]. The percentage of hamstring injury prevention after performing NHE during pre-training also varied between studies, including 65\%-70\% [10], 57\%-72\% [1], and 51\% [11]. The elite European soccer premier league has reported a poor effect with NHE, with only $11 \%$ of hamstring injury prevention rate in randomized controlled trials [12].

Recently, adding post-training to pre-training FIFA 11+ prevention program as a cooling down program to the normal pre-training FIFA $11+$ as a warming up program has been found to be able to decrease injury risk by $82 \%$ for all lower limb injuries with only $85 \%$ of compliance rate to the program [11]. In 2017, it was recommended that future research including medical staff was needed to conduct more effective studies on injury prevention with a high compliance rate [5]. Since adding post-training to FIFA $11+$ prevention program was effective [11], it was found that only post-training Nordics (completed in a fatigued state) could also result in increase of fascicle angle of pennation (an increase of $2.14^{\circ}$ ) and fascicle muscle length (an increase of $0.2 \mathrm{~cm}$ ) after 12 weeks of training [1]. However, adding NHE as a post-training ex- ercise program besides the normal pre-training one has not been reported yet. To fill such gap in the literature, the main aim of this study was to determine the effect of pre-training and post-training NHE in decreasing hamstring injury rates and severity.

\section{MATERIALS AND METHODS}

The study design was a randomized controlled trial conducted in the summer football season in 2018. From five total football teams in Alexandria, Egypt, Sporting club was randomly chosen by simple randomization. Thirty-four male active professional football players who were normal and healthy were enrolled for this study (Fig. 1). Inclusion criteria were as follows: (1) male players with normal healthy conditions; (2) aged between 21 and 35 years with weight range of $60-86 \mathrm{~kg}$, height range of $1.65-1.95 \mathrm{~m}$, and body mass index range of $22-22.9 \mathrm{~kg} / \mathrm{m}^{2}$; (3) being an active player; and (4) free of any hamstring injury at least for 6 months before applying the protocol. A power analysis was performed to confirm that the sample size was adequate. At $95 \%$ confidence interval

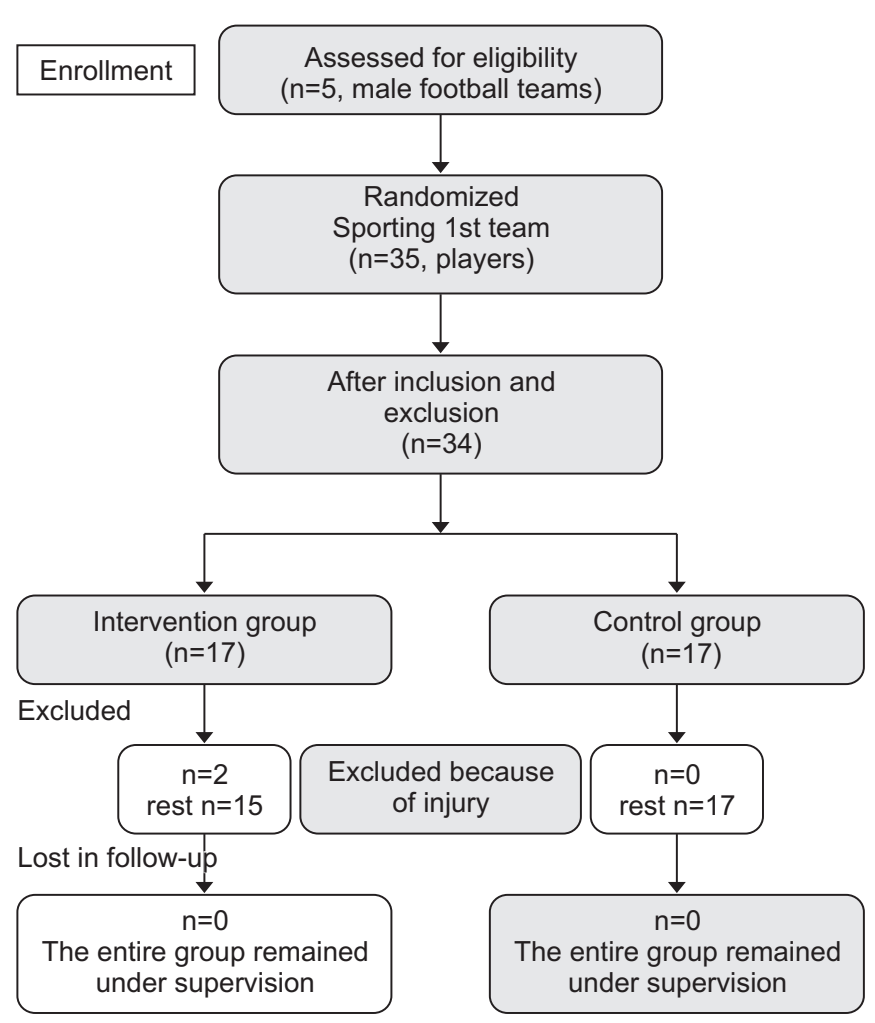

Fig. 1. Flowchart of this study. 
(CI), the sample size was calculated to be 34 subjects with the power of the study of $80.0 \%$. Before enrollment in this study, each player signed an informed consent according to our Faculty Ethical Committee. This study was registered in Pan African Control Trial (Approval ID: PACTR201810754318796).

Players were excluded if they participated in a team after the start of the trial or any previous lower extremity injury that required medical attention in the past 6 months. Players with any systemic disease, cardiovascular disease, neurological disorders, or any surgery in the previous year were also excluded from this study.

Players were randomly assigned to two equal groups using opaque sealed envelopes. Group one included 17 players who performed NHE both during pre-training and post-training. Group two included 17 players who performed NHE only during pre-training. Both groups performed traditional warming up and cooling down protocols. Hamstring injury rate of the team was recorded using football (soccer) injury form. Results were compared to data of the club of the previous season (control group) after 3 months (full protocol). Injury incidence rate and injury risk ratio (IRR) were calculated for each group.

\section{Treatment procedures}

The protocol duration was 12 weeks. NHE was performed twice per week except for the first week which was performed once. The pre-training NHE protocol (Table 1) followed that of Lovell et al. [1]. The post-training NHE

Table 1. Nordic hamstring exercise during pre-training

\begin{tabular}{rcccc}
\hline Week & $\begin{array}{c}\text { Session } \\
\text { frequency }\end{array}$ & Sets & Repetitions & $\begin{array}{c}\text { Weekly } \\
\text { volume }\end{array}$ \\
\hline 1 & 1 & 2 & 5 & 10 \\
2 & 2 & 3 & 5 & 30 \\
\hline 3 & 2 & 3 & 6 & 36 \\
\hline 4 & 2 & 4 & 6 & 48 \\
\hline 5 & 2 & 4 & 7 & 56 \\
\hline 6 & 2 & 4 & 7 & 56 \\
\hline 7 & 2 & 4 & 8 & 64 \\
\hline 8 & 2 & 4 & 8 & 64 \\
\hline 9 & 2 & 4 & 9 & 72 \\
10 & 2 & 4 & 9 & 72 \\
11 & 2 & 4 & 10 & 80 \\
12 & 2 & 4 & 12 & 96 \\
\hline
\end{tabular}

protocol is shown in Table 2.

\section{Nordic hamstring exercise}

Player started in a kneeling position with the torso from knees held upward, rigid, and straight. The therapist ensured that the player's feet were in contact with the ground throughout the exercise by sitting in her both knees and applying pressure to the player's heels/lower legs as shown in Fig. 2. Instruction was given to the player to lower his upper body to the ground as slowly as possible to maximize loading in the eccentric phase. Hands and arms were used to break his forward fall and to push him back up after his chest had touched the ground to minimize loading in the concentric phase as shown in Fig. 3.

\section{Warming up and cooling down}

The traditional warming up Brazilian exercises [13] for 20 minutes in the form of high knee jumps, arm swings, faster high knees, side stepping, upper body rotations, run pace, and counter jumps were performed by all participants enrolled in this study. The normal cooling down was done by cold water immersion for 10 minutes [14]. Low-intensity aerobic running was chosen as mode of active recovery from the exercise [15].

\section{Outcome measures}

The primary outcome of this study was hamstring injury incidence as initial and recurrent injuries. Injury incidence was reported in absolute numbers as well as injury

Table 2. Nordic hamstring exercise during post-training

\begin{tabular}{ccccc}
\hline Week & $\begin{array}{c}\text { Session } \\
\text { frequency }\end{array}$ & Sets & Repetitions & $\begin{array}{c}\text { Weekly } \\
\text { volume }\end{array}$ \\
\hline 1 & 1 & 1 & 2 & 5 \\
2 & 2 & 1 & 2 & 10 \\
\hline 3 & 2 & 1 & 3 & 12 \\
4 & 2 & 2 & 3 & 24 \\
\hline 5 & 2 & 2 & 3 & 28 \\
6 & 2 & 2 & 3 & 28 \\
\hline 7 & 2 & 2 & 4 & 32 \\
\hline 8 & 2 & 2 & 4 & 32 \\
\hline 9 & 2 & 2 & 4 & 36 \\
10 & 2 & 2 & 5 & 36 \\
\hline 11 & 2 & 2 & 5 & 40 \\
12 & 2 & 2 & 6 & 48 \\
\hline
\end{tabular}




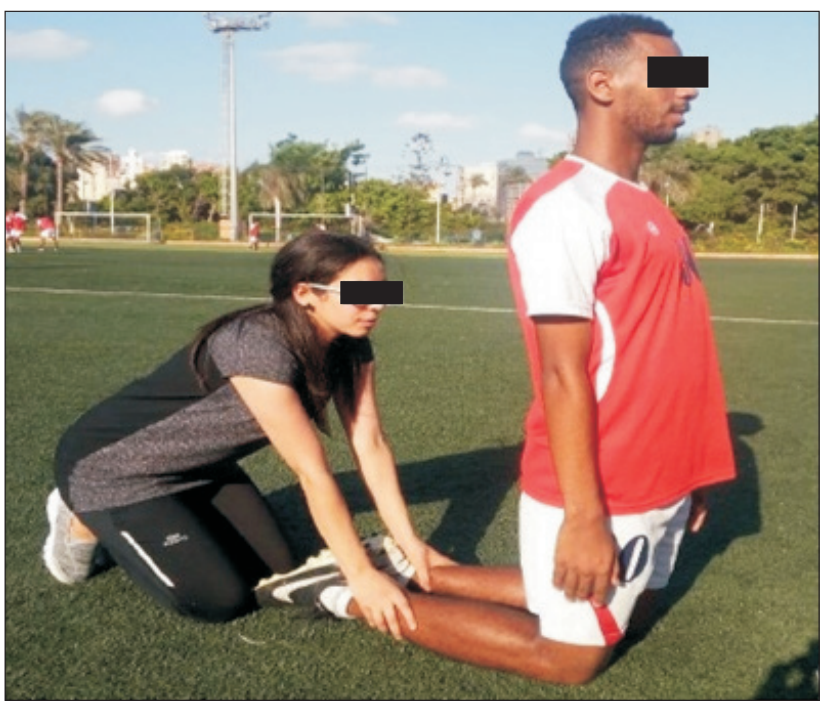

Fig. 2. Illustrating therapist and patient's starting position during Nordic hamstring exercise.

incidence rate (number of injuries per 1,000 player hours in matches and training). Secondary outcomes were injury severity and compliance in both groups. Injury severity was defined as the number of days lost from the injury date till full return to training and being available for match selection: It included slight (0 days), minimal (1-3 days), mild (4-7 days), moderate (8-28 days), severe (>28 days), and career ending levels. Compliance was calculated according to players' rate of participation.

\section{Data analysis}

Data were analyzed using IBM SPSS software package version 20.0 (IBM, Armonk, NY, USA). Qualitative data were described using number and percentage. Chisquare test was used to compare different groups regarding categorical variables to assess the effect of intervention on injury incidence and injury severity. Quantitative data were described using mean and standard deviation for normally distributed data. For normally distributed data, comparison between two independent populations was done using independent t-test while more than two populations were analyzed using F-test (ANOVA). Significance test results were quoted as two-tailed probabilities. Significance of obtained results was judged at 5\% level.

Hamstring injury incidence was counted from data collected using the Australian football (soccer) association injury form. Injury incidence rate was calculated by dividing the number of incident injuries by the total time

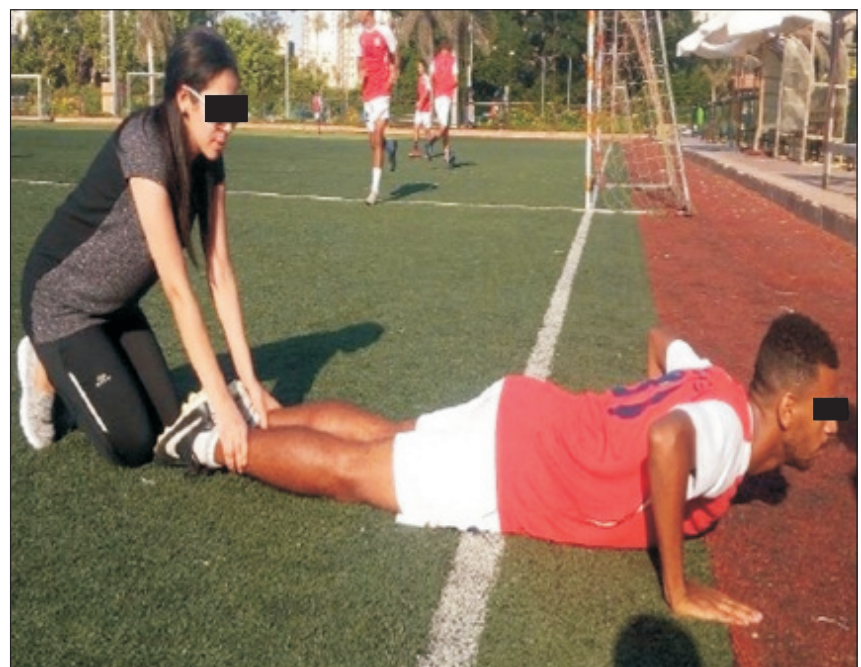

Fig. 3. Illustrating ending position of the Nordic hamstring exercise.

at risk and multiplied by 1,000 as this could accommodate variations in exposure time of individual athletes as shown in the following equation:

Injury incidence rate $=\frac{\text { Number of incidence injuries }}{\text { Total time at risk }} \times 1,000$,

while IRR, a new standard for analyzing the efficacy of injury prevention programs [8], was calculated using the following equation [5].

$$
\operatorname{IRR}=\frac{\text { Injury incidence in intervention group one }}{\text { Injury incidence in intervention group two }} .
$$

An IRR $<1$ indicates a positive intervention effect. For example, an IRR of 0.30 represents a $70 \%$ reduction. Individual injury rates and IRR along with CI were determined. Compliance with the intervention protocol was calculated based on information provided on player's adherence to the program for both groups (groups one and two). As the protocol had 50 sessions for group one and 25 sessions for group two, compliance was calculated as $[10,16]$ :

Compliance (\%) for group one $=\frac{\text { Amount of NHE sessions performed }}{50} \times 100$,

Compliance $(\%)$ for group two $=\frac{\text { Amount of NHE sessions performed }}{25} \times 100$. 


\section{RESULTS}

Thirty-four professional male football players with mean age of $24.3 \pm 3.8$ years old were assigned into the two groups (17 players each). All of them completed the designated full protocol except two players due to injuries causing absence for more than a week. Baseline characteristics of these players including age, weight, height, body mass index, and leg dominance were collected and analyzed with t-test and chi-square test, showing no significant difference between the two groups (all p>0.05), meaning that they were homogenous groups as shown in Table 3.

Table 3. Demographic data of subjects in the two interventions groups

\begin{tabular}{llcc}
\multicolumn{1}{c}{ Item } & $\begin{array}{c}\text { Intervention } \\
\text { group one }\end{array}$ & $\begin{array}{c}\text { Intervention } \\
\text { group two }\end{array}$ & p-value \\
\hline Age $(\mathrm{yr})$ & $24.2 \pm 3.2$ & $24.5 \pm 4.4$ & 0.412 \\
BMI $\left(\mathrm{kg} / \mathrm{m}^{2}\right)$ & $22.4 \pm 0.4$ & $22.5 \pm 0.2$ & 0.251 \\
Height $(\mathrm{m})$ & $1.80 \pm 0.10$ & $1.90 \pm 0.11$ & 0.093 \\
Weight $(\mathrm{kg})$ & $74.6 \pm 7.2$ & $77.8 \pm 6.0$ & 0.083 \\
Leg dominance & & & \\
Right & $16(94.1)$ & $15(88.2)$ & 0.744 \\
\hline Left & $1(5.9)$ & $2(11.8)$ & \\
\hline
\end{tabular}

Values are presented as mean \pm standard deviation or number (\%).

\section{Exposure time}

During the 12 weeks of program, players of both groups had average exposure time to training sessions and playing matches. Group one had an average exposure time of $116.3 \pm 13.2$ hours and group two had an average exposure time of 117.6 \pm 5.7 hours. By t-test, there was no significant $(p>0.05)$ difference in average exposure time between the two groups at $95 \% \mathrm{CI}(0.65-3.2)$.

Effects of NHE on whole injury incidence, initial injury, injury recurrence, and injury severity are summarized in Table 4. When compared to results of the whole past team regarding initial injuries, recurrent injuries, and severity of injuries using chi-square test, initial injuries between the two intervention groups and the control group (previous season) at $\chi^{2}=8.25$ and $95 \%$ CI (0.21-0.74) revealed that $35.1 \%$ were injured which was highly significant $(\mathrm{p}<0.05)$. In the control group, $18.9 \%$ suffered recurrent hamstring injuries. The percentage of recurrent injuries was significantly higher than in the two interventional groups: $18.9 \%$ were injured at $\chi^{2}=6.58$ with $95 \% \mathrm{CI}$ (0.012-0.651) and at ANOVA test $(\mathrm{F}=12.65)$ with $95 \% \mathrm{CI}$ (0.104-0.92). Regarding the severity of previous team's injures, the number of absent days ranged 5-10 days, with a mean of 7.95 days. This was significantly higher than the severity of injury in the two interventional groups $(\mathrm{p}=0.001)$.

Table 4. Comparing the effect of NHE on whole injury incidence, initial injury, injury recurrence, and injury severity among the three groups of this study

\begin{tabular}{|c|c|c|c|}
\hline & Intervention group one & Intervention group two & $\begin{array}{c}\text { Control group } \\
\text { (previous season) }\end{array}$ \\
\hline NHE & $\begin{array}{l}\text { Pre- and post-training } \\
\text { NHE (50 total sessions) }\end{array}$ & $\begin{array}{l}\text { Pre-training NHE only } \\
\text { (25 total sessions) }\end{array}$ & No NHE \\
\hline $\begin{array}{l}\text { Total risk time }^{\mathrm{a}} \\
\text { (exposure hour) }\end{array}$ & $116.3 \pm 13.2$ & $117.6 \pm 5.7$ & $117 \pm 5.5$ \\
\hline $\begin{array}{l}\text { Reported initial } \\
\text { hamstring injuries }\end{array}$ & $\begin{array}{l}1 \text { injury }(5.9 \%) \text { out of } 17 \\
\text { players }\end{array}$ & $\begin{array}{l}3 \text { injuries }(17.6 \%) \text { out of } \\
17 \text { players }\end{array}$ & $\begin{array}{c}13 \text { injuries }(35.1 \%) \\
\text { out of } 35 \text { players }\end{array}$ \\
\hline $\begin{array}{l}\text { Reported recurrent } \\
\text { hamstring injuries }\end{array}$ & None & $\begin{array}{l}1 \text { injury (5.9\%) out of } \\
17 \text { players }\end{array}$ & $\begin{array}{r}7 \text { injuries (18.9\%) } \\
\text { out of } 35 \text { players }\end{array}$ \\
\hline Total injuries & $1(5.9 \%)$ & $4(23.5 \%)$ & $20(62.5 \%)$ \\
\hline Severity of injury & 1 day & 2-3 days & 5-10 days \\
\hline Prevention percentage & 92.3 & 77 & 0 \\
\hline resulted & \multicolumn{2}{|c|}{70 (the whole program) } & - \\
\hline
\end{tabular}

NHE, Nordic hamstring exercise.

${ }^{a}$ Total training and playing time. 


\section{Compliance to protocol}

Only two players were excluded from the intervention group due to injury leading to absence more than a week. All other players full completed the whole program (50 sessions for intervention group one and 25 sessions for intervention group two). The compliance percentage was the highest among studies in the same area of research. It was calculated based on information provided by the researcher. It was $100 \%$ for intervention group two, $98.7 \%$ for intervention group one with a total compliance rate of $99.3 \%$, which was the highest compliance rate that was ever achieved.

IRR

Using the following equations [5]:

Injury incidence rate $=\frac{\text { Number of incidence injuries }}{\text { Total time at risk }} \times 1,000$,

$$
\mathrm{IRR}=\frac{\text { Injury incidence in intervention group one }}{\text { Injury incidence in intervention group two }},
$$

the two studied groups resulted in totally injury prevention percentage of $70 \%$ than the previous season. With only intervention group one, it showed an injury prevention rate of $92.3 \%$. Also, there was a $85 \%$ decrease in recurrence rates with intervention group two compared to previous season and a $100 \%$ prevention rate with intervention group one.

\section{DISCUSSION}

This randomized control trial was conducted to investigate a highly effective eccentric hamstring strength training in the form of Nordic hamstring exercise during both pre-training and post-training or pre-training only to reduce incidence rates of hamstring initial injuries and recurrent injuries and their severity for professional male soccer players and compare it to the previous season when NHE was not applied in any prevention program. Until 2018, hamstring injuries were still considered as number 1 muscle injury in football. These injuries were increasing in the last decade with high recurrences, increasing personal suffering for players despite all preventive measures were applied with increased knowledge about this muscle injury [17].
The Arabic questionnaire survey for implementation strategies of injury prevention programs was used to detect the adoption of Egyptian coach to prevention protocols in the previous season which was considered a limitation of this study [2]. The study was controlled as much as possible for baseline characteristics of players including age, weight, height, body mass index, and leg dominance. By applying t-test and chi-square test, there was no significant difference between the two groups ( $p>0.05$ ), meaning that they were homogenous groups. Also, the average exposure time to training sessions and playing matches of the players of the two studied groups was 116.3 \pm 13.2 hours total for the intervention group one and 117.6 \pm 5.7 hours total for the intervention group two. By applying t-test, there was no significant difference ( $p>0.05)$ between the two groups at 95\% CI (0.65-3.2). Such similarity in exposure time provides information that it is not a contributing factor to incidence of injuries.

With these limitations, results of this study revealed that the there was a significant increase in whole injury in intervention group two than that in intervention group one. The intervention group one had only 1 (5.9\%) injury while the intervention group two had $4(23.5 \%)$ injuries $(\mathrm{p}<0.05)$. However, there was no significant difference in hamstring muscle initial injury at the end of the intervention protocol between the two groups $(\mathrm{p}=0.287)$.

These results were in agreement with a recent study published in 2018 [18], showing that NHE was very effective even with the shortest protocol done. However, in the present study, the intervention protocol's duration was 12 weeks reported to be a sufficient duration for muscular adaptation to prevent hamstring injuries [5]. This result could be because intervention group one had higher eccentric strength by performing Nordic hamstring during both pre-training and post-training, meaning they were able to withstand the high lengthening force on muscle fibers as it developed tension and contracts to control the movement performed at late swing and pre-stance phases known to be the highest injury exposure phases [19].

Results of the current study were in line with those of another study reporting that fascicle muscle length and fascicle angle of pennation were increased about $0.2 \mathrm{~cm}$ and $2.14^{\circ}$, respectively, after applying NHE during posttraining rather than applying it during pre-training [1]. Their increases are very critical in sprinters and soccer players to decrease injury rates [20]. Furthermore, these 
results were in agreement with a previous [17] showing that pre-training only was insufficient for hamstring muscle injuries prevention.

For initial and recurrent injury comparison, chi-square test revealed that there was no statistically significant difference between the two groups. When comparing initial and recurrent injuries of the two NHE groups with the team in the previous season, results revealed significant difference. For the team in the previous season, $35.1 \%$ had initial injuries and $\mathbf{1 8 . 9 \%}$ had recurrent injuries. These were significantly higher those in the two NHE groups (intervention groups one and two). There was also a significant increase in the severity of injury in the team during the previous season than the two NHE groups $(\mathrm{p}<0.01)$. The number of days absent for the previous team ranged 5-10 days, with a mean of 7.95 days. However, it was 1 day for intervention group one and 2.7 days for intervention group two. This result was in agreement with Lovell et al. [1] reporting that muscle thickness and fascicle angle of pennation increased more when NHE was applied during post-training rather than during pre-training. This may also illustrate the no recurrence of hamstring injury in the intervention group one compared to previous team which had 7 recurrent hamstring injuries more than once.

The two studied groups resulted in totally injury prevention percentage of $70 \%$ than the previous season and $92.3 \%$ prevention compared to only intervention group one. Also, there was a $85 \%$ decrease in recurrence rates with intervention group two compared to the previous season and $100 \%$ prevention with intervention group one. These results were higher than all previous studies applied in that area of research. Results were calculated using $[5,11]$ :

Injury incidence rate $=\frac{\text { Number of incidence injuries }}{\text { Total time at risk }} \times 1,000$,

$$
\text { IRR }=\frac{\text { Injury incidence in intervention group one }}{\text { Injury incidence in intervention group two }} .
$$

In 2015, van der horst et al. [10] performed a study and found a total of $\mathbf{3 8}$ hamstring injuries were affecting 36 (6.2\%) of 579 players. The risk for hamstring injuries was significantly $(\mathrm{p}=0.005)$ reduced in the intervention group compared to that in the control group. However, injury severity was not significantly different between the intervention group and the control group. Compliance rate with the intervention protocol was $91 \%$. However, they performed NHE during pre-training only [10]. In 2018, van der Horst [17] found that pre-training only was not enough to prevent injury.

Compliance or adherence to the applied protocol has been mentioned as a very important factor affecting results obtained and effectiveness of the protocol $[9,11]$. The efficacy of pre- and post-training FIFA 11+ injury prevention program was related to the degree of compliance. The greater the compliance, the greater the reduction in injuries $[2,11]$. In the present study, only two players were excluded from the intervention group due to injury resulting in absence for more than a week. All other players fully completed the whole program (50 sessions for intervention group one and 25 sessions for intervention group two). Compliance rate was calculated based on information provided by the researcher. Finally, it was found to be $100 \%$ for intervention group two, $98.7 \%$ for intervention group one, with total compliance rate of $99.3 \%$. This was the highest compliance rate ever achieved among all studies conducted in this area of research.

According to these results, a similar study should be conducted with a large number of football teams to provide a wide representation of data. Other studies should also be performed with different professional levels to provide more evidence for exercise effectiveness in different levels. Studies on female football team are also needed using objective tools for assessing injury and measuring peak muscle torque. In addition, the relationship between hamstring injuries and other lower limb injuries merits further study.

In conclusion, the use of NHE as a prevention protocol for professional soccer players was successful in reducing the number of both initial and recurrent hamstring muscle injuries as well as their severity. In addition, we found that using NHE during both pre-training and posttraining had the greatest effect in preventing hamstring injuries.

\section{CONFLICT OF INTEREST}

No potential conflict of interest relevant to this article was reported. 


\section{ACKNOWLEDGMENTS}

The authors would like to thank Dr. David RodriguezSanz, professor and researcher of the Department of Physiotherapy and Podiatry, Faculty of Sports Science, European University of Madrid, Villaviciosa de Odón, Spain and Facultad de Enfermería, Fisioterapia y Podologia, Universidad Complutense de Madrid, Madrid, Spain for his useful contributions to the writing of this manuscript.

\section{AUTHOR CONTRIBUTION}

Conceptualization: Elerian AE. Methodology: Elerian AE, El-Sayyad MM, Dorgham HA. Formal analysis: Elerian AE, El-Sayyad MM. Project administration: Elerian AE, Dorgham HA. Writing - original draft: Elerian AE. Writing - review and editing: Elerian AE, El-Sayyad MM. Approval of final manuscript: all authors.

\section{REFERENCES}

1. Lovell R, Knox M, Weston M, Siegler JC, Brennan S, Marshall PW. Hamstring injury prevention in soccer: before or after training? Scand J Med Sci Sports 2018;28:658-66.

2. Al Attar WS, Soomro N, Sinclair PJ, Pappas E, Muaidi QI, Sanders RH. Implementation of an evidencebased injury prevention program in professional and semi-professional soccer. Int J Sports Sci Coach 2018;13(1):113-21.

3. van Beijsterveldt AM, van de Port IG, Vereijken AJ, Backx FJ. Risk factors for hamstring injuries in male soccer players: a systematic review of prospective studies. Scand J Med Sci Sports 2013;23:253-62.

4. Wan X, Qu F, Garrett WE, Liu H, Yu B. The effect of hamstring flexibility on peak hamstring muscle strain in sprinting. J Sport Health Sci 2017;6:283-9.

5. Al Attar WS, Soomro N, Sinclair PJ, Pappas E, Sanders RH. Effect of injury prevention programs that include the Nordic hamstring exercise on hamstring injury rates in soccer players: a systematic review and metaanalysis. Sports Med 2017;47:907-16.

6. Seymore KD, Domire ZJ, DeVita P, Rider PM, Kulas AS. The effect of Nordic hamstring strength training on muscle architecture, stiffness, and strength. Eur J Appl
Physiol 2017;117:943-53.

7. Bourne MN, Opar DA, Williams MD, Al Najjar A, Shield AJ. Muscle activation patterns in the Nordic hamstring exercise: Impact of prior strain injury. Scand J Med Sci Sports 2016;26:666-74.

8. Al Attar WS, Soomro N, Pappas E, Sinclair PJ, Sanders RH. How effective are F-MARC injury prevention programs for soccer players? A systematic review and meta-analysis. Sports Med 2016;46:205-17.

9. Steffen K, Emery CA, Romiti M, Kang J, Bizzini M, Dvorak J, et al. High adherence to a neuromuscular injury prevention programme (FIFA 11+) improves functional balance and reduces injury risk in Canadian youth female football players: a cluster randomised trial. Br J Sports Med 2013;47:794-802.

10. van der Horst N, Smits DW, Petersen J, Goedhart EA, Backx FJ. The preventive effect of the Nordic hamstring exercise on hamstring injuries in amateur soccer players: a randomized controlled trial. Am J Sports Med 2015;43:1316-23.

11. Al Attar WSA, Soomro N, Pappas E, Sinclair PJ, Sanders RH. Adding a post-training FIFA 11+ exercise program to the pre-training FIFA 11+ injury prevention program reduces injury rates among male amateur soccer players: a cluster-randomised trial. J Physiother 2017;63:235-42.

12. Arnason A, Andersen TE, Holme I, Engebretsen L, Bahr R. Prevention of hamstring strains in elite soccer: an intervention study. Scand J Med Sci Sports 2008;18:40-8. 13. Ayala F, Calderon-Lopez A, Delgado-Gosalbez JC, Parra-Sanchez S, Pomares-Noguera C, HernandezSanchez S, et al. Acute effects of three neuromuscular warm-up strategies on several physical performance measures in football players. PLoS One 2017;12:e0169660.

14. Machado AF, Ferreira PH, Micheletti JK, de Almeida AC, Lemes IR, Vanderlei FM, et al. Can water temperature and immersion time influence the effect of cold water immersion on muscle soreness? A systematic review and meta-analysis. Sports Med 2016;46:503-14.

15. Meyer T, Wegmann M, Poppendieck W, Fullagar $\mathrm{HH}$. Regenerative interventions in professional football. Sports Orthop Traumatol 2014;30:112-8.

16. Thorborg K, Krommes KK, Esteve E, Clausen MB, Bartels EM, Rathleff MS. Effect of specific exercisebased football injury prevention programmes on the 
overall injury rate in football: a systematic review and meta-analysis of the FIFA 11 and $11+$ programmes. $\mathrm{Br}$ J Sports Med 2017;51:562-71.

17. van der Horst N. Preventing hamstring injuries in football through enhanced exercise and RTP strategies. Br J Sports Med 2018;52:684-5.

18. Ribeiro-Alvares JB, Marques VB, Vaz MA, Baroni BM. Four weeks of Nordic hamstring exercise reduce muscle injury risk factors in young adults. J Strength Cond
Res 2018;32:1254-62.

19. Van Hooren B, Bosch F. Is there really an eccentric action of the hamstrings during the swing phase of highspeed running? Part I. A critical review of the literature. J Sports Sci 2017;35:2313-21.

20. Guex K, Degache F, Morisod C, Sailly M, Millet GP. Hamstring architectural and functional adaptations following long vs. short muscle length eccentric training. Front Physiol 2016;7:340. 